\title{
Mucin 1, a signal transduction membrane- bound mucin, is present in human disc tissue and is downregulated in vitro by exposure to IL-1ß or TNF- $\alpha$
}

Helen E. Gruber ${ }^{1,2^{*}}$, Jane A. Ingram ${ }^{1}$, Gretchen L. Hoelscher ${ }^{1}$, Emilio Marrero ${ }^{1}$ and Edward N. Hanley Jr ${ }^{1}$

\begin{abstract}
Background: Back pain and disc degeneration have a growing socioeconomic healthcare impact. Mucin 1 (MUC1) is a transmembrane glycoprotein whose extracellular and intracellular domains participate in cellular signaling. Little is currently known about the presence or role of MUC1 in human disc degeneration.

Methods: In this IRB-approved research study, 29 human disc specimens were analyzed for MUC1 immunohistochemical localization and gene expression, and annulus fibrosus (annulus) cells were also isolated and cultured in 3D. Microarray analysis assessed expression levels of MUC1 in healthy and degenerated disc tissue and in cells exposed to proinflammatory cytokines (IL-1ß or TNF- $\mathrm{a}$ ).

Results: MUC1 was shown to be present in annulus cells at the protein level using immunochemistry, and its expression was significantly upregulated in annulus tissue from more degenerated grade $V$ discs compared to healthier grade $1-I I$ discs $(p=0.02)$. A significant positive correlation was present between the percentage of MUC1-positive cells and disc grade $(p=0.009)$. MUC1 expression in annulus cells cultured in 3D was also analyzed following exposure to IL-1ß or TNF-a; exposure produced significant MUC1 downregulation ( $p=0.0006)$.

Conclusions: Here we present the first data for the constitutive presence of MUC1 in the human disc, and its altered expression during disc degeneration. MUC1 may have an important role in disc aging and degeneration by acting as a regulator in the hypoxic environment, helping disc cells to survive under hypoxic conditions by stabilization and by activation of HIF-1a as previously recognized in pancreatic cancer cells.
\end{abstract}

Keywords: Mucin 1, Intervertebral disc, Disc degeneration, Interleukin 1-ß, Tumor necrosis factor-a

\section{Background}

Mucin 1 (MUC1) is a transmembrane glycoprotein with a protein backbone of 20 amino acid residues repeated in tandem, a transmembrane sequence, and a 69 amino acid-long tail [4]. This conserved tail received posttranslational modifications from growth factor receptor tyrosine kinases (RTKs), and can participate in interactions with factors which can directly influence transcriptional regulatory ability [3]. In non-polarized cells,

\footnotetext{
* Correspondence: helen.gruber@carolinashealthcare.org

${ }^{1}$ Department of Orthopaedic Surgery, Carolinas Medical Center, PO Box 32861, Charlotte, NC, USA

${ }^{2}$ Orthopaedic Research Biology, Carolinas Medical Center, Cannon Research

Center, Room 304, PO Box 32861, Charlotte, NC 28232, USA
}

MUC1 can make contact with the RTKs via its tail, and can then signal in response to growth and differentiation factors. In the tumor microenvironment, MUC1 signaling has been found to reprogram transcription of connective tissue growth factor (CTGF, also called CCN2), which is a strong mediator of extracellular matrix (ECM) remodeling and angiogenesis [3]. Tran et al. have recently suggested that the specialized cross-talk between CTGF and hypoxia inducible factor-1 (HIF-1) may be important in the avascular adult degenerating disc [22]. Stabilized expression HIF-1 $\alpha$ is now recognized as one of the "healthy nucleus [pulposus] phenotypic markers" [7, 17], and knockout of HIF-1 $\alpha$ has been shown to accelerate disc degeneration in a mouse model 
[24]. Chaika et al. have shown that MUC1 can facilitate recruitment of HIF-1 $\alpha$ in an hypoxia-dependent manner, suggesting that MUC1 can act as an important regulator in the hypoxic environment, helping cells to survive under hypoxic conditions by its stabilization and activation of HIF- $1 \alpha$ [5].

Because of the potential importance of MUClin disc biology and degeneration, in the present work we searched for expression of MUC1 in human disc tissue and its immunohistochemical localization; we also tested for its expression when annulus fibrosus (annulus) cells cultured in 3D were exposed to either interleukin 1-beta (IL-1ß) or tumor necrosis factor-alpha (TNF- $\alpha$ ), two proinflammatory cytokines that are well-recognized in disc degeneration $[14,15]$. These proinflammatory cytokines can be bound in the extracellular matrix surrounding cells within the degenerating disc, resulting in higher localized regional concentrations [16].

\section{Methods}

\section{Clinical study population}

Experimental study of human disc specimens was approved in a prospective manner by the authors' Human Subjects Institutional Review Board at Carolinas Medical Center. Informed consent was waived by the ethical board because disc tissue used in the present study was removed as part of routine surgical practice. As we have reported previously, our scoring of disc degeneration utilized a modified Thompson scoring system which incorporated author ENH's radiologic, MRI and surgical findings. The Thompson disc grading system scores disc degeneration across the span of disc degeneration, from healthy discs (Thompson grade I) to discs with the most advanced degeneration (grade $\mathrm{V}$, the most severe stage of degeneration) [21]. As we have reported previously, specimens from patients were derived from surgical procedures performed on individuals with herniated discs and degenerative disc disease. These specimens were transported to the laboratory in sterile tissue culture medium. Control non-surgical donor disc specimens were obtained via the National Cancer Institute Cooperative Human Tissue Network (CHTN); these specimens were shipped overnight to the laboratory in sterile tissue culture medium and processed as described below. Procurement of these control non-surgical specimens was included in our approved Institutional Review Board protocol.

\section{Gene expression analyses of MUC1 in human disc tissue and in cultured human annulus cells Human disc tissue}

As we have previously described, human disc tissue (Table 1) was snap frozen in liquid nitrogen, pulverized (BioPulverizer, BioSpec Products, Inc., Bartlesville, OK,
USA), and homogenized via the FastPrep-24 instrument (MP Biomedicals L.L.C., Santa Ana, CA, USA). Total RNA $(100 \mu \mathrm{g})$ was then harvested, reverse transcribed, amplified, labeled, fragmented and hybridized to the Affymetrix human U133 X3P microarray chips. We utilized the GCOS Affymetrix GeneChip Operating System (version 1.2, Affymetrix, Santa Clara, CA 95051) to determine gene expression levels of MUC1.

\section{Cultured cells}

Annulus cells were established in monolayer culture [9], and expanded for use in 3D in a collagen sponge as previously described [13]. Cells were cultured over14 days in 3D with media changes using either control conditions (minimal essential medium plus 20\% FBS) or experimental test conditions with addition of IL-1beta $\left(10^{-2} \mathrm{pM}\right)$ or TNF-alpha $\left(10^{3} \mathrm{pM}\right)$ (Table 1$)$. Doses used were previously determined in our laboratory $[8,10]$. As we have previously reported, RNA was harvested from cells at experiment conclusion and gene expression studies carried out using microarray analysis. Total RNA $(100 \mu \mathrm{g})$ was harvested, reverse transcribed, amplified, labeled, fragmented and hybridized to the Affymetrix human U133 X3P microarray chips. The GCOS Affymetrix GeneChip Operating System (version 1.2, Affymetrix, Santa Clara, CA 95051) was used for determining gene expression levels of MUC1 (gene identifier Hs.89603.6.A1_3p_a_at).

\section{Statistical analysis of microarray data}

Statistical analyses utilized GeneSifterTM web-based software to analyze microarray data. Employing the GCRMA (Robust multi-array average) method, Affymetrix ".cel" files were uploaded to the GeneSifterTM web site, normalized, and corrected for false discovery rate (FDR). As we have previously reported, statistical significance was determined using Student's t-test (2 tailed, unpaired) and significance was set at $p<0.05$ ). Fold change was set at 2.0. Additional statistical analyses were performed using unpaired t-tests and Mann-Whitney tests (GraphPad Instat 3, GraphPad Software, Inc., San Diego, CA). If data were not distributed normally, analyses utilized nonparametric statistical methods.

\section{Immunolocalization of MUC1}

Paraffin sections from human discs or from cells cultured in 3D were cut at $4 \mu \mathrm{m}$ (Table 1), collected on PLUS slides (Cardinal Health, Dublin, $\mathrm{OH}$ ) and dried at $60{ }^{\circ} \mathrm{C}$. Sections were deparaffinized using xylene (Cardinal) and then rehydrated through graded alcohols (AAPER, Shelbyville, KY) to distilled water. $3 \% \mathrm{H}_{2} \mathrm{O}_{2}$ (Sigma, St Louis, MO) was used to block endogenous peroxidase. Sections were incubated for one hour with anti-MUC-1 (epithelial membrane antigen MA5-13168, 
Table 1 Demographic features for subjects whose disc tissue was evaluated with immunohistochemistry or whose tissue or cells were utilized for molecular analysis

\begin{tabular}{|c|c|c|c|c|c|}
\hline Subject number & Age & Thompson score & Vertebral level & Specimen type ${ }^{a}$ & Experimental use $^{\mathrm{b}}$ \\
\hline 1 & 34 & 1 & Lumbar & CHTN & $\mathrm{Mol}$ \\
\hline 2 & 30 & $\|$ & L3-4 & CHTN & Mol \\
\hline 3 & 30 & $\|$ & L3-4 & CHTN & $\mathrm{Mol}$ \\
\hline 4 & 54 & $\|$ & $\llcorner 4-5$ & Surgical specimen & $\mathrm{Mol}$ \\
\hline 5 & 21 & $\|$ & L5-S1 & Surgical specimen & Mol \\
\hline 6 & 40 & $\|$ & $\llcorner 4-5$ & Surgical specimen & Mol \\
\hline 7 & 41 & V & $\llcorner 4-5$ & Surgical specimen & Mol \\
\hline 8 & 57 & V & C6-7 & Surgical specimen & Mol \\
\hline 9 & 72 & V & L4-S1 & Surgical specimen & Mol \\
\hline 10 & 1 & । & Lumbar & CHTN & Imm \\
\hline 11 & Newborn & । & Lumbar & CHTN & Imm \\
\hline 12 & 21 & $\|$ & L5-S1 & Surgical specimen & Imm \\
\hline 13 & 24 & $\|$ & L5-S1 & Surgical specimen & Imm \\
\hline 14 & 41 & $\|$ & L5-S1 & Surgical specimen & Imm \\
\hline 15 & 29 & III & L5-S1 & Surgical specimen & Imm \\
\hline 16 & 53 & III & L5-S1 & Surgical specimen & Imm \\
\hline 17 & 54 & III & $\llcorner 4-5$ & Surgical specimen & Imm \\
\hline 18 & 58 & III & L2-3 & Surgical specimen & Imm \\
\hline 19 & 39 & IV & $\llcorner 4-5$ & Surgical specimen & Imm \\
\hline 20 & 56 & IV & L2-3 & Surgical specimen & Imm \\
\hline 21 & 59 & IV & L2-3 & Surgical specimen & Imm \\
\hline 22 & 78 & IV & L3-4 & Surgical specimen & Imm \\
\hline 23 & 39 & V & L5-S1 & Surgical specimen & Imm \\
\hline 24 & 56 & V & L5-S1 & Surgical specimen & Imm \\
\hline 25 & 62 & V & L5-S1 & Surgical specimen & Imm \\
\hline 26 & 59 & IV & $\llcorner 4-5$ & Surgical specimen & 3D and $\mathrm{Mol}$ \\
\hline 27 & 59 & IV & $\llcorner 4-5$ & Surgical specimen & 3D and $\mathrm{Mol}$ \\
\hline 28 & 57 & III & $C 4-5$ & Surgical specimen & 3D and Mol \\
\hline 29 & 54 & IV & L3-4 & Surgical specimen & $3 \mathrm{D}$ and $\mathrm{Mol}$ \\
\hline
\end{tabular}

${ }^{a} C H T N$ Cooperative Human Tissue Network, normal donor specimen, $O R$ surgical specimen, $L$ lumbar, $C$ cervical

${ }^{\mathrm{b}} / \mathrm{mm}$ immunohistochemistry on disc tissue, 3D and Mol cells cultured in 3D and 3D mRNA harvested for microarray analysis, Mol human annulus tissue mRNA harvested for microarray analysis

ThermoFisher, Rockford, IL) at a 1:200 dilution. The secondary reagent utilized was Vector Immpress Reagent, Anti-Mouse Ig (Vector Laboratories, Burlingame, CA) for 30 minutes followed by DAB (Dako) for 5 minutes. Slides were rinsed in water, counterstained with light green, dehydrated, cleared and mounted with resinous mounting media. Mouse IgG (Dako, Carpenteria, CA) was used as a negative control; human colon tissue was used as a positive control.

The number of cells positive for immunolocalization of MUC1 was determined and the percentage of positive cells analyzed for its relationship with the Thompson grade of that disc tissue. The mean number of cells scored/specimen was $209 \pm 51$ (13) (mean \pm S.D. (n)) and the range 149 to 295. Standard statistical methods were performed using GraphPad Instat 3 (GraphPad Software, Inc., San Diego, CA). Spearman's correlation coefficient was calculated to test for the association of Thompson grade with the \% of cells positive for Muc1 immunolocalization, and $\mathrm{r}^{2}$ was used to determine goodness of fit.

\section{Results}

Table 1 describes the source of disc tissue for specimens utilized for immunolocalization of MUC1, for cell culture experiments, and for tissue gene expression studies.

Positive MUC1 localization at the cell membrane was present in many spindle-shaped and round cells in the 
annulus (Fig. 1a, b, and d). There were fewer positive cells noted in nucleus specimens (Fig. 1e). (Figure 1f presents a representative image from a negative control specimen.) In some instances, annulus cells showed a more general cytoplasmic localization pattern (Fig. 1c). In sites where there was a clear separation of the cells from the surrounding ECM, localization could be seen along the margin of the pericellular matrix (Fig. 1d). No localization was present in the territorial or interterritorial matrix of the annulus, or within the nucleus matrix. The percentage of cells positive for MUC1 localization was determined and statistically assessed for a relationship with disc grade; a positive significant correlation was present $\left(r^{2}=0.473, p=0.009\right)$, indicating that disc grade accounted for $47.3 \%$ of variation in the fraction of cells with MUC1 localization (Fig. 2).
Examination of molecular expression of MUC1 in human annulus tissue showed a small, but significant 1.2 fold upregulation in more degenerated, grade $\mathrm{V}$ discs compared to expression in healthier grade I and II discs $(p=0.02$; Fig. 3; Table 1). No correlation was identified between the stages of disc degeneration and MUC1 levels in disc tissue.

In vitro studies were carried to test the effect of exposure to proinflammatory cytokines on MUC1 expression. Human annulus cells were cultured in a 3D microenvironment for 14 days during which they were exposed to either control conditions or doses of IL-1beta $\left(10^{-2} \mathrm{pM}\right)$ or TNF-alpha $\left(10^{3} \mathrm{pM}\right)$. Dose levels had been optimized in previous studies [10, 12]. Immunohistochemistry identified MUC1 localization in many cells in the 3D construct under control (Fig. 4a), IL-1ß-treated cells
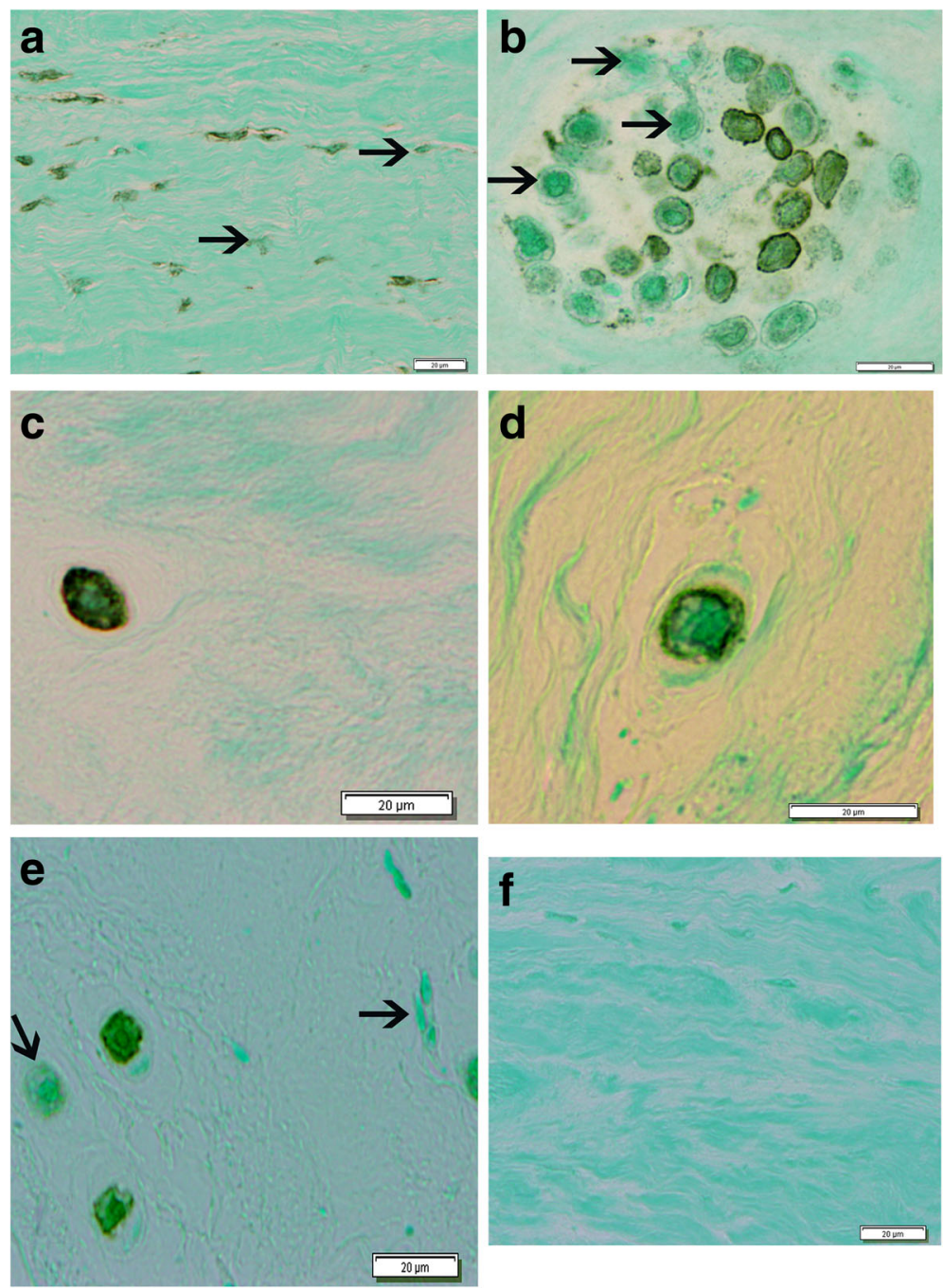

Fig. 1 Representative images showing immunolocalization of MUC1. a Grade IV specimen shows the outer annulus, with many spindle-shaped cells. b Large cluster of cells in the inner annulus showing cells with positive or negative localization. c Cytoplasmic immunolocalization present in an inner annulus cell from a grade IV specimen. $\mathbf{d}$ Localization along the rim of the lacunar margin of a disc cell in the inner annulus. e Fewer immunopositive cells were present in the nucleus pulposus regions of the disc. $\mathbf{f}$ Negative control section adjacent to that shown in a. (Arrows mark cells which do not show localization. Bar $=20 \mu \mathrm{m}$ ) 


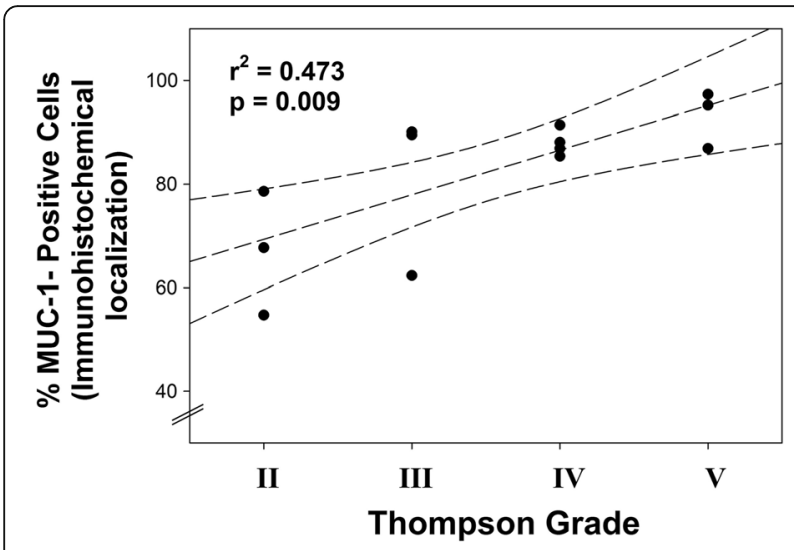

Fig. 2 A significant, positive correlation was present between the proportion of cells exhibiting MUC1 immunolocalization and the disc Thompson grade $\left(r^{2}=0.473 ; p=0.009\right)$. (Dashed lines show the $95 \%$ confidence interval for the correlation)

(Fig. 4b) and in TNF- $\alpha$-treated cells (Fig. 4c). (Figure 4d presents a representative negative control). Molecular analyses showed highly significant down regulation of MUC1 expression in cells exposed to IL-1ß or TNF- $\alpha$ $(p=0.0006$; Fig. 5).

\section{Discussion}

The present studies appear to be the first investigations of MUC1 in the human disc. MUC1 has previously been identified on the apical cell surface of epithelial cells, and is overexpressed in human carcinomas and hematologic malignancies in which it binds to caspase- 8 and FADD, blocks caspase- 8 recruitment, and thus prevents activation of the death receptor-induced extrinsic apoptotic pathway in cancer cells [2].

MUC1 is a surface-associated mucin with recognized signal transduction activity. Singh and Hollingsworth have recently reviewed the ways by which both the MUC1

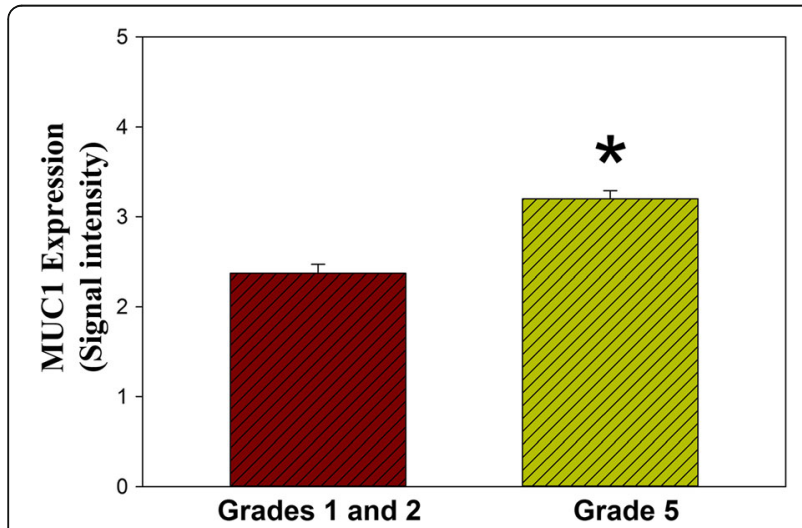

Fig. 3 Graphical presentation of the significant increase in the relative MUC1 expression in vivo in more degenerated Thompson grade $V$ disc tissue vs. healthier grade I and II discs ( $p=0.02$; from Mann-Whitney evaluation)

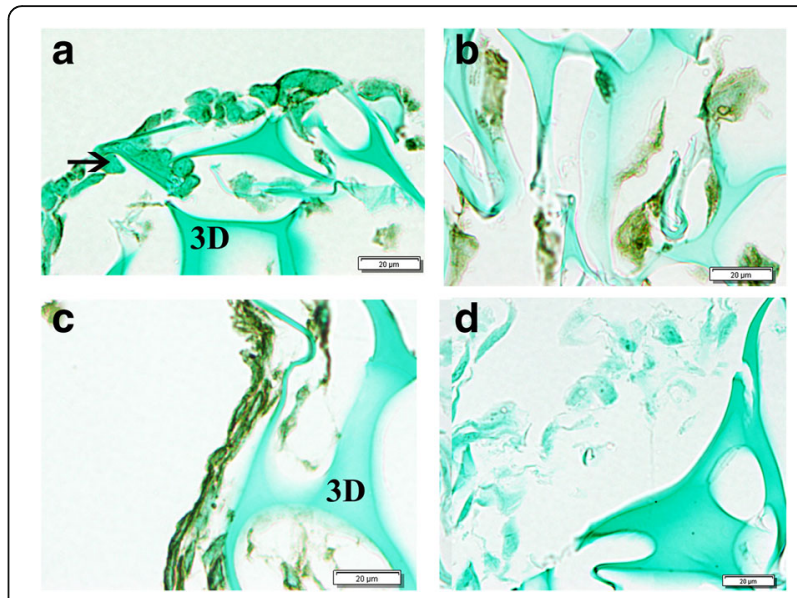

Fig. 4 Representative images of cells cultured in 3D with immunolocalization of MUC1. a. Cells in the 3D construct under control; b. IL-1ß-treated cells; c. TNF-a-treated cells. "3D" marks the collagen sponge framework in which cells were cultured.

d. Representative image from a negative control. (Arrows mark cells which do not show localization. Bar $=10 \mu \mathrm{m})$

extracellular and intracellular domains participate in cell signaling [20]. MUC1 has roles in several important signaling pathways, including Ras, ß-catenin, p120 catenin, p53, and estrogen receptor $\alpha[19,23]$. MUC1 may play an important part in the avascular disc by mechanisms modulating the effects of hypoxia signaling through HIF transcription factors. This MUC1 activity has been studied by Chaika et al. in pancreatic cancer cells, and the authors noted that MUC1 could be acting either by regulating HIF stability and it interaction partners, or by direct signaling through the MUC1 cytoplasmic tail using direct interaction with transcription factors $[5,20]$.

Data presented here showed an upregulation of MUC1 in more degenerated Thompson grade $\mathrm{V}$ compared to healthier grade I and II discs. In agreement with this was the positive correlation between the percentage of MUC1-

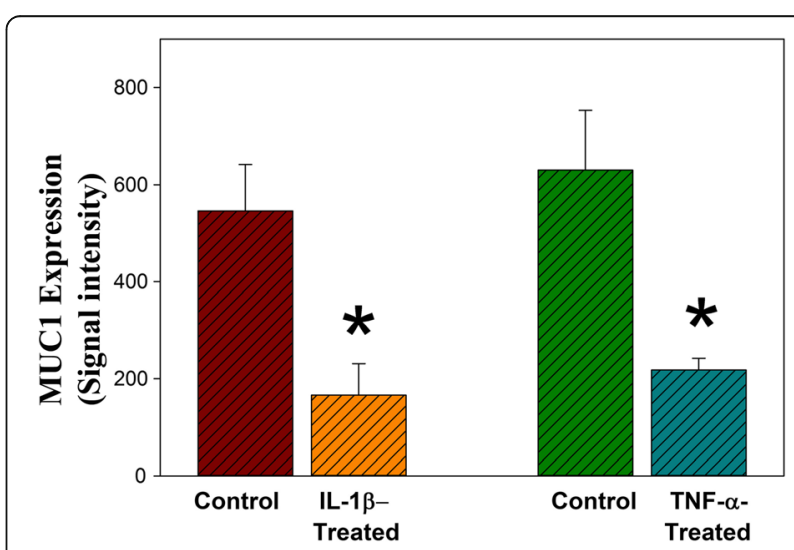

Fig. 5 Graphical presentation of the relative MUC1 expression in control annulus cells compared to IL-1-B or TNF-a treated cells cultured in $3 \mathrm{D}\left({ }^{*} p=0.0006\right.$ vs. relative control) 
positive cells and disc grade $(p=0.009)$; the high $r^{2}$ value for this correlation suggests that disc grade accounted for $47.3 \%$ of variation in the fraction of cells with MUC1 immunolocalization.

Our findings of the significant downregulation of MUC1 during IL-1 $ß$ and TNF- $\alpha$ exposure also suggest potential selective involvement of MUC1 during changes in the proinflammatory disc milieu during degeneration. As shown in the recent review by Risbud and Shapiro, we now know that there are numerous cytokines, in addition to IL- $1 \beta$ and TNF- $\alpha$, which undergo important changes during the process of disc degeneration; these include IL-1 $\alpha$, IL-6 and IL-17 (see [18] for a recent review). Our novel findings here with MUC1 and its increasing expression during disc degeneration point to the importance of future work which focuses upon interactions of MUC1 with major cytokines and chemokines in the degenerating disc.

Oxidative stress-induced apoptosis is also an important event in the aging and degenerating disc which puts an already small cell population at further risk for a decrease in cell numbers [11]. Both in vivo and in vitro research has focused on the modulation of apoptosis by MUC1 expression [20]. Studies have shown that MUC1 positive cells were more sensitive to apoptosis induced by FasL compared to MUC1 negative cells; the proposed mechanism was upregulation of the cell surface Fas receptor by increased intracellular trafficking [6].

As previously noted, MUC1 signaling in tumors has been found to reprogram transcription of connective tissue growth factor CTGF, which is a strong mediator of extracellular matrix (ECM) remodeling and angiogenesis [3]. In the present work, we identified a significant upregulation in MUC1 expression in annulus tissue from more degenerated grade $\mathrm{V}$ discs compared to healthier grade I-II discs $(p=0.02)$. Abbot et al. have previously shown that the stage of degeneration of the nucleus pulposus can influence the nucleus cell response to CTGF [1], suggesting another potentially important role for MUC1 in annulus cells.

Unfortunately, we were unable to carry out qRT-PCR molecular studies on cells/tissue described here because of insufficient amounts of remaining mRNA. Future research on the role of MUC1 in disc aging and degeneration appears warranted to determine whether MUC1 might act as a regulator in the hypoxic environment, helping disc cells to survive under hypoxic conditions by stabilization and activation of HIF-1 $\alpha$ (as shown in pancreatic cancer cells by Chaika et al. [5], or by exerting an influence on the response to CTGF. We look forward to future studies which should include qRT-PCR gene expression analysis, and an expanded exploration of the relationship of MUC1 to disc aging and degeneration.

\section{Conclusion}

This is the first study describing the constitutive presence of MUC1 in the human disc, and its increased expression during disc degeneration, and its in vitro downregulation during exposure to the proinflammatory cytokines IL-1ß and TNF- $\alpha$. Since MUC1 may have an important role in disc aging and degeneration by acting as a regulator in the hypoxic environment (helping disc cells to survive under hypoxic conditions by stabilization and by activation of HIF- $1 \alpha$ as previously recognized in pancreatic cancer cells), we look forward to future studies on the role of MUC1 in disc aging and degeneration.

\section{Abbreviations \\ CHTN: Cooperative Human Tissue Network; CTGF: Connective tissue growth factor, also called CCN2; HIF-1: Hypoxia inducible factor-1; IL-1ß: Interleukin-1 beta; MUC1: Mucin 1; TNF-a: Tumor necrosis factor-alpha}

\section{Acknowledgements}

The authors wish to thank Nury Steuerwald, Ph.D. and Judy Parsons in the Molecular Biology Core for expert technical assistance with microarray analysis, Synthia Bethea for assistance in cell culture.

\section{Funding}

The authors wish to acknowledge the support of the Brooks Back Pain Research Endowment for general laboratory support used in this work.

\begin{abstract}
Availability of data and materials
The datasets supporting the conclusions presented in this article are available at https://www.ncbi.nlm.nih.gov/. Information can found within the following datasets: GSE41883 (TNF-a); GSE27494 (IL-1ß), and GSE23130 (Disc Tissue). Some of the more recent molecular data sets no longer provided access to raw data due to changes of our institutional licensing agreements with these companies. We will be happy to share available data with researchers following their individual requests.
\end{abstract}

\section{Authors' contributions}

HEG and ENH made substantial contributions to the conception and design of this study and participated in design and coordination of manuscript preparation. EM and GLH made substantial contributions to acquisition of molecular data and analysis and interpretation of these data. molecular analyses. HEG drafted and revised the manuscript. JAI made substantial contributions to immunohistochemical procedures and acquisition of that data. All authors made substantial contributions to the methods development of their respective responsibilities and also reviewed and approved the final manuscript.

\section{Competing interests}

The authors declare that they have no competing interests.

\section{Ethics approval and consent to participate}

The protocol for the study was approved by our Institutional Review Board of the Carolinas HealthCare System under the research project entitled "Characterization of gene expression and cell-extracellular matrix interactions during intervertebral disc aging and degeneration", IRB file \# 08-04-09E, valid until April 5, 2017. The need for informed consent was waived by the ethical board since disc tissue was removed as part of routine surgical practice.

Consent for publication

Not applicable.

\section{Publisher's Note}

Springer Nature remains neutral with regard to jurisdictional claims in published maps and institutional affiliations. 
Received: 29 August 2016 Accepted: 28 April 2017

Published online: 08 May 2017

\section{References}

1. Abbott RD, Purmessur D, Monsey RD, Brigstock DR, Laudier DM, latridis JC. Degenerative grade affects the responses of human nucleus pulposus cells to Link-N, CTGF, and TGFß3. J Spinal Disord Tech. 2013;26:E86-94.

2. Agel J, Ahmad R, Kawano T, Raina D, Kharbanda S, Kufe D. MUC1 oncoprotein blocks death receotpr-mediated apoptosis by inhibiting recruitment of caspase-8. Cancer Res. 2008;68:6136-44.

3. Behrens ME, Grandgenett PM, Bailey JM, Singh PK, Yi C-H, Yu F, et al. The reactive tumor microenvironment: $\mathrm{MUC1}$ signaling directly reprograms transcription of CTGF. Oncogene. 2010:29:5667-77.

4. Cao Y, Blohm B, Ghadimi BM, Stosiek P, Xing P-X, Karsten U. Mucins (MUC1 and MUC3) of gastrointestinal and breast epithelia reveal different and heterogeneous tumor-associated abberations in glycosylation. J Histochem Cytochem. 1997:45:1547-57.

5. Chaika NV, Gebregiworgis T, Lewallen ME, Purohit V, Radhakrishnanm P, Liu X, et al. MUC1 mucin stabilizes and activates hypoxia-inducible factor 1 alpha to regulate metabolism in pancreatic cancer. Proc Natl Acad Sci U S A. 2012;109: 133787-13792.

6. Chaturvedi R, Srivastava RK, Hisatsune A, Shankar S, Lillehoj EP, Kim KC. Augmentation of Fas ligand-induced apoptosis by MUC1 mucin. Internatl J Oncol. 2005;26:1169-76.

7. Gogate SS, Nasser R, Shapiro IM, Risbud MV. Hypoxic regulation of beta-1,3glucuronyltransferase 1 expression in nucleus pulposus cells of the rat intervertebral disc: role of hypoxia-inducible factor proteins. Arthritis Rheum. 2011;63:1950-60.

8. Gruber HE, Marrero E, Ingram JA, Hoelscher GL, Hanley EN Jr. Constitutive expression of IL-22 in the human intervertebral disc and its reduction by exposure to pro-inflammatory cytokines in vitro. Biotech Histochem. 2017. In Press.

9. Gruber HE, Fisher Jr EC, Desai B, Stasky AA, Hoelscher G, Hanley EN. Human intervertebral disc cells from the annulus: Three- dimensional culture in agarose or alginate and responsiveness to TGF- $\beta 1$. Exp Cell Res. 1997;235:13-21.

10. Gruber HE, Hoelscher GL, Bethea S, Hanley EN. Interleukin 1-beta upregulates brain-derived neurotrophic factor, neurotrophin 3 and neuropilin 2 gene expression and NGF production in annulus cells. Biotech Histochem. 2012;87:506-11.

11. Gruber HE, Hoelscher GL, Bethea S, Hanley EN. Mitochondrial membrane potential and nuclear and gene expression changes during human disc cell apoptosis. In vitro and in vivo annulus findings. Spine. 2015;40:876-82.

12. Gruber HE, Hoelscher GL, Ingram JA, Morton DS, Hanley EN Jr. Human annulus cells regulate PAPP-A and IGFBP-4 expression, and thereby insulinlike growth factor bioavailability, in response to proinflammatory cytokine exposure in vitro. Conn Tiss Res. 2013:54:432-8.

13. Gruber HE, Hoelscher GL, Leslie K, Ingram JA, Hanley EN Jr. Three-dimensiona culture of human disc cells within agarose or a collagen sponge: assessment of proteoglycan production. Biomaterials. 2006;27:371-6.

14. Hoyland JA, Le Maitre C, Freemont AJ. Investigation of the role of IL-1 and TNF in matrix degradation in the intervertebral disc. Rheumatol. 2008;47:809-14.

15. LeMaitre $\mathrm{CL}$, Hoyland JA, Freemont AJ. Catabolic cytokine expression in degenerate and herniated human intervertebral discs: IL-1ß and TNFa expression profile. Arthritis Res Ther. 2007:9/4:R77

16. Merline $R$, Schaefer RM, Schaefer $L$. The matricellular function of small leucine-rich proteoglycans (SLRPs). J Cell Commun. 2009:3:323-35.

17. Risbud MV, Schoepflin ZR, Mwale F, Kandel RA, Grad S, latridis JC, et al. Defining the phenotype of young healthy nucleus pulposus cells: recommendations of the Spine Research Interest Group at the 2014 annual ORS meeting. J Orthop Res. 2015;33:283-93.

18. Risbud MV, Shapiro JR. Role of cytokines in intervertebral disc degeneration: pain and disc content. Nat Rev Rheumatol. 2014;10:44-56.

19. Singh PK, Behrens ME, Eggers JP, Cerny RL, Bailey JM, Shanmugam K, et al. Phosphorylation of MUC1 by Met modulates interaction with p53 and MMP1 expression. J Biol Chem. 2008;283:26985-95.

20. Singh PK, Hollingsworth MA. Cell surface-associated mucins in signa transduction. Trends Cell Biol. 2006;16:467-76.
21. Thompson JP, Pearce RH, Schechter MT, Adams ME, Tsang IKY, Bishop PB. Preliminary evaluation of a scheme for grading the gross morphology of the human intervertebral disc. Spine. 1990;15:411-5.

22. Tran CM, Shapiro IM, Risbud MV. Molecular regulation of CCN2 in the intervertebral disc: Lessons learned form other connective tissues. Matrix Biol. 2013;32:298-306.

23. Wei $X, X u H$, Kufe D. Human mucin 1 oncoprotein represses transcription of teh p53 tumor suppressor gene. Cancer Res. 2007;67:1853-8.

24. Wu WJ, Zhang XK, Zheng XF, Yang YJ, Jiang SD, Jiang LS. SHH-dependent knockout of HIF-1 alpha accelerated the degenerative process in mouse intervertebral disc. Internatl J Immunopathol Pharmacol. 2013;26:601-9.

\section{Submit your next manuscript to BioMed Central and we will help you at every step:}

- We accept pre-submission inquiries

- Our selector tool helps you to find the most relevant journal

- We provide round the clock customer support

- Convenient online submission

- Thorough peer review

- Inclusion in PubMed and all major indexing services

- Maximum visibility for your research

Submit your manuscript at www.biomedcentral.com/submit 